Louisiana State University

LSU Digital Commons

8-1-2017

\title{
Stabilization and robustness analysis for time-varying systems with time-varying delays using a sequential subpredictors approach
}

\author{
Frédéric Mazenc \\ Laboratoire des Signaux et Systèmes \\ Michael Malisoff \\ Louisiana State University
}

Follow this and additional works at: https://digitalcommons.Isu.edu/mathematics_pubs

\section{Recommended Citation}

Mazenc, F., \& Malisoff, M. (2017). Stabilization and robustness analysis for time-varying systems with time-varying delays using a sequential subpredictors approach. Automatica, 82, 118-127. https://doi.org/ 10.1016/j.automatica.2017.04.020

This Article is brought to you for free and open access by the Department of Mathematics at LSU Digital Commons. It has been accepted for inclusion in Faculty Publications by an authorized administrator of LSU Digital Commons. For more information, please contact ir@lsu.edu. 
archives-ouvertes

\section{Stabilization and Robustness Analysis for Time-Varying Systems with Time-Varying Delays using a Sequential Subpredictors Approach*}

Frédéric Mazenc, Michael Malisoff

\section{To cite this version:}

Frédéric Mazenc, Michael Malisoff. Stabilization and Robustness Analysis for Time-Varying Systems with Time-Varying Delays using a Sequential Subpredictors Approach *. Automatica, Elsevier, 2017, 82, pp.118-127. 10.1016/j.automatica.2017.04.020 . hal-01660103

\section{HAL Id: hal-01660103 \\ https://hal.inria.fr/hal-01660103}

Submitted on 9 Dec 2017

HAL is a multi-disciplinary open access archive for the deposit and dissemination of scientific research documents, whether they are published or not. The documents may come from teaching and research institutions in France or abroad, or from public or private research centers.
L'archive ouverte pluridisciplinaire HAL, est destinée au dépôt et à la diffusion de documents scientifiques de niveau recherche, publiés ou non, émanant des établissements d'enseignement et de recherche français ou étrangers, des laboratoires publics ou privés. 


\title{
Stabilization and Robustness Analysis for Time-Varying Systems with Time-Varying Delays using a Sequential Subpredictors Approach*
}

\author{
Frédéric Mazenc $^{\mathrm{a}}$ and Michael Malisoff ${ }^{\mathrm{b}}$ \\ ${ }^{a}$ EPI DISCO Inria-Saclay, Laboratoire des Signaux et Systèmes, CNRS, CentraleSupélec, Université Paris-Sud, \\ 3 rue Joliot Curie, 91192, Gif-sur-Yvette, France. \\ ${ }^{\mathrm{b}}$ Department of Mathematics, Louisiana State University, Baton Rouge, LA 70803-4918, USA.
}

\begin{abstract}
We provide a new sequential predictors approach for the exponential stabilization of linear time-varying systems. Our method circumvents the problem of constructing and estimating distributed terms in the control laws, and allows arbitrarily large input delay bounds, pointwise time-varying input delays, and uncertainties. Instead of using distributed terms, our approach to handling longer delays is to increase the number of predictors. We obtain explicit formulas to find lower bounds for the number of required predictors. The formulas involve bounds on the delays and on the derivatives of the delays. We illustrate our method in three examples.
\end{abstract}

Key words: Delay, robustness, stability, time-varying

\section{Introduction}

This work continues our search (which we began in Mazenc and Malisoff (2017)) for predictive control methods for time-varying systems that can be applied without computing Lie derivatives and without computing distributed terms, and which can compensate for arbitrarily long input delays. Our work is motivated by the ubiquity of input delays across engineering, coupled with the challenges that one may encounter when building delay tolerant feedback controls, if one applies traditional emulation or prediction methods that can involve distributed terms. See, e.g., Bekiaris-Liberis and Krstic (2013), Michiels and Niculescu (2007), and Richard (2003) for overviews on delay compensating control, Sharma et al. (2011) for constant electromechanical input delays in muscle response in neuromuscular electrical stimulation (or NMES), and Kamalapurkar et al. (2016) and Merad

\footnotetext{
^ Corresponding author: F. Mazenc. A summary of some of this work (with only a sketch of a proof of the theorem) appeared in the proceedings of the 55th IEEE Conference on Decision and Control; see the end of Section 1 for a comparison between the present work and the conference version. Malisoff was supported by NSF-ECCS Grant 1408295, and by Roy Paul Daniels Professorship \#3 in the LSU College of Science.
}

Email addresses:

frederic.mazenc@12s. centralesupelec.fr (Frédéric Mazenc), malisoff@lsu.edu (Michael Malisoff). et al. (2016) for extensions to NMES under time-varying input delays.

For constant coefficient linear systems, it often suffices to use linear matrix inequalities (or LMIs) to build delay tolerant controls, but many important linear systems are time-varying. For instance, when tracking reference trajectories and linearizing around the reference trajectories, we obtain time-varying linear systems, even if the original system is time invariant. See Mazenc et al. (2014), and Germani et al. (2002) for systems with delayed outputs, which lead to input delayed systems when the output for one system is an input for another system.

Traditional input delay compensation methods can roughly be grouped into three approaches. One approach is to solve a stabilization problem with the input delay set equal to 0 , and to then look for upper bounds on the input delay that the resulting closed loop system can tolerate, without sacrificing the desired stability properties. Two advantages of this so-called emulation approach are that (a) it makes it possible to use relatively simple controls for undelayed systems (such as Lie derivative feedbacks and other approaches from Khalil (2002)) and (b) the strict Lyapunov functions that one obtains from solving the feedback design problem for the corresponding undelayed system can often be transformed into Lyapunov-Krasovskii functionals, which can in turn be used to compute bounds on the input delays that the closed loop system can tolerate. 
See Fridman and Niculescu (2008) for background on Lyapunov-Krasovskii functionals, and Mazenc et al. (2008) for ways to transform strict Lyapunov functions for undelayed systems into Lyapunov-Krasovskii functionals for the corresponding input delayed systems.

Another approach is the reduction model method, where the control is expressed implicitly as a solution of an integral equation, which can lead to challenging problems of numerically computing controls, especially when the system has uncertainty or the delay is time-varying. Prediction is another useful method, where the states in the controls are replaced by numerical predictions of the states. Standard prediction or reduction model methods can compensate for arbitrarily long input delays, and so may have an advantage over emulation for communication networks or multi-agent problems that are prone to long input delays, but the distributed terms in their controls (which use all values of the input or the state on some interval of times) may make them harder to implement; see Artstein (1982), Bekiaris-Liberis and Krstic (2012), Mazenc et al. (2012), and Witrant et al. (2007).

This paper provides a new sequential predictors approach to exponential stabilization of time-varying linear systems with time-varying input delays, and therefore builds on recent notable works such as Najafi et al. (2013) (which used LMI methods for time invariant linear systems to build sequential predictors) and Léchappé (2015) (which extended Najafi et al. (2013) by studying constant coefficient linear systems with time-varying delays, which is a smaller class of systems than the time-varying systems that we consider here). It also extends Mazenc and Malisoff (2017), which was confined to constant delays. Since we do not use any distributed terms or Lie derivatives in our control, our work is also very different from the classical reduction model or the more recent prediction approaches that have been used by M. Krstic and others (as in Bresch-Pietri and Petit (2014), Karafyllis and Krstic (2013), and Krstic (2009)).

Our approach uses several dynamic extensions. Each dynamic extension has the same dimension as the original system. This contrasts with the reduction model approach, where the integral equation that produces the control has the same dimension as the control. Since we do not use distributed terms, our work differs from AhmedAli et al. (2016), Ahmed-Ali et al. (2013), and other works that use several dynamic extensions and distributed terms. We obtain closed form control formulas and ways to compute lower bounds on the number of required extensions. Our work is mainly a theoretical and methodological development. However, we illustrate our work in three examples, including a pendulum dynamics that we studied in Mazenc et al. (2014), where we used a reduction model approach and distributed controls but did not cover time-varying delays.

There are several other notable works that use prediction without using distributed terms, but which do not co- ver the problems that we solve in the present work. The works Ahmed-Ali et al. (2012), Cacace et al. (2016c), Cacace et al. (2016a), and Zhou et al. (2012) focused on time-invariant linear systems $\dot{x}=A x+B u$, and they use eigenvalue conditions on $A$ and bounds on the delays or controllability conditions, without guaranteeing robustness under uncertainty; strict feedback systems were covered in Cacace et al. (2016b) by adding conditions on the coefficient matrices of a new system that is obtained by using a diffeomorphic transformation, but we do not require such conditions here; Cacace et al. (2014b) proved asymptotic stability results with prescribed decay rates for linear time-invariant systems by using partial spectrum assignment; Cacace et al. (2014c) was also limited to time-invariant linear systems; Germani et al. (2002) used a globally drift-observability condition (which we also do not require here) to cover nonlinear systems; and Zhou (2014a) and Zhou (2014b) gave sufficient conditions for stabilizability for time-varying linear systems under pseudo-predictor feedback with an integral delay system that is also not needed in the present work. Our work is also reminiscent of Cacace et al. $(2014 a)$, which is devoted to chain observers for time invariant nonlinear systems with time-varying measurement delays, and so does not cover the uniform global exponential stabilization results that we present here.

We use standard notation and definitions. Throughout the sequel, the dimensions are arbitrary, unless otherwise noted. We omit arguments of functions when they are clear, and we assume that the initial times $t_{0}$ for our solutions of our systems are $t_{0}=0$, but we can write analogs for general choices of $t_{0} \geq 0$. We use $|\cdot|$ to denote the usual Euclidean norm and the induced matrix norm, $|\phi|_{\infty}$ (resp., $|\phi|_{\mathcal{I}}$ ) is the essential supremum (resp., supremum over any interval $\mathcal{I}$ ) for any bounded measurable function $\phi$, and $I_{n}$ is the $n \times n$ identity matrix.

Our preliminary version Mazenc and Malisoff (2016) of this work only provides a sketch of the proof of its main result, while here we provide a complete and new proof, and a new example from identification theory that was not in Mazenc and Malisoff (2016). Our new proof includes a new Lyapunov-Krasovskii functional approach that can allow smaller values for the number of required sequential subpredictors than what was required in Mazenc and Malisoff (2016); see Remark 4.

\section{Main Result}

We study systems of the form

$$
\dot{x}(t)=A(t) x(t)+B(t) u(t-h(t))+\delta(t),
$$

where the state $x$ and the control $u$ are valued in $\mathbb{R}^{n}$ and $\mathbb{R}^{\ell}$, respectively, $h: \mathbb{R} \rightarrow[0, \infty)$ is a known time-varying delay, and $\delta:[0, \infty) \rightarrow \mathbb{R}^{n}$ is an unknown measurable essentially bounded function representing unmodeled features or actuator errors. We make two assumptions: 
Assumption 1 The function $h$ is $C^{1}$ and bounded from above by a constant $c_{h}>0, \dot{h}$ has a finite lower bound $\underline{h} \in \mathbb{R}, \dot{h}$ is bounded from above by a constant $l_{h} \in(0,1)$, and $\dot{h}$ has a global Lipschitz constant $n_{h}>0$.

Assumption 2 The functions $A$ and $B$ are bounded and continuous, and there is a known bounded continuous function $K:[0, \infty) \rightarrow \mathbb{R}^{\ell \times n}$ such that

$$
\dot{x}(t)=[A(t)+B(t) K(t)] x(t)
$$

is uniformly globally exponentially stable on $\mathbb{R}^{n}$ to 0 .

Assumption 1 can model many delays, e.g., by using a standard denseness argument to arbitrarily closely approximate many non- $C^{1}$ delays, including discontinuous delays; see Remark 2 . In terms of an integer $m>1$ that we specify later, we use the functions

$$
\Omega_{i}(t)=t-\frac{i}{m} h(t) \text { and } \theta_{j}(t)=\Omega_{m-j+1}^{-1}\left(\Omega_{m-j}(t)\right)
$$

for all $i \in\{0, \ldots, m\}$ and $j \in\{1, \ldots, m\}$, and define

$$
R_{1}=\dot{\theta}_{1} \text { and } R_{i}(t)=\dot{\theta}_{i}(t) R_{i-1}\left(\theta_{i}(t)\right) \text { for } i>1 \text {. }
$$

The preceding functions are used to define the coefficients in our subpredictors and to produce the required exponential decay estimate of our transformed error vector in our theorem. Such functions exist because our upper bounds $c_{h}$ and $l_{h} \in(0,1)$ from Assumption 1 imply that the $\Omega_{i}$ 's have the range $\mathbb{R}$ and are strictly increasing. Hence, the $\theta_{i}$ 's are also strictly increasing and $C^{1}$. The inverses in (3) can be computed numerically using standard programs, e.g., the command

$$
\mathrm{g}=\text { InverseFunction }[\text { Function }[\mathrm{t}, \mathrm{t}-\mathrm{h}[\mathrm{t}] / \mathrm{m}]]
$$

in Mathematica (2015) to compute $\Omega_{1}^{-1}(t)$ for a known choice of $h(t)$. We also assume that the initial functions are constant on $(-\infty, 0]$. In terms of the constants

$$
u_{c}=n_{h} \frac{c_{h}}{\left(1-l_{h}\right)^{2}}+\frac{l_{h}}{1-l_{h}}
$$

and

$$
\begin{aligned}
& b_{1}=\left[1+\left(1+\frac{u_{c}}{m}\right)^{m}|A|_{\infty}\right]\left(1+\frac{u_{c}}{m}\right)^{m}|A|_{\infty}, \\
& b_{2}=\left[1+\left(1+\frac{u_{c}}{m}\right)^{m}|A|_{\infty}\right]^{2}, \text { and } \\
& b_{3}=b_{2}\left(1+\frac{u_{c}}{m}\right),
\end{aligned}
$$

we then prove:

Theorem 1 If Assumptions 1-2 hold, and if the constant integer $m \geq 2$ satisfies

$$
2\left(\frac{c_{h}}{\left(1-l_{h}\right) m}\right)^{2}\left(b_{1}^{2}+4 b_{3}^{2}\right)<1
$$

then we can find positive constants $\mu_{1}$ and $\mu_{2}$ such that for all solutions of (1) in closed loop with the control

$$
u(t)=K(t+h(t)) z_{m}(t)
$$

where $z_{m}$ is the last $n$ components of the system

$$
\begin{aligned}
\dot{z}_{1}(t)= & R_{1}(t) A\left(\theta_{1}(t)\right) z_{1}(t) \\
& +R_{1}(t) B\left(\theta_{1}(t)\right) u\left(\Omega_{m-1}(t)\right) \\
& +L_{1}(t)\left[z_{1}\left(\theta_{1}^{-1}(t)\right)-x(t)\right] \\
\dot{z}_{i}(t)= & R_{i}(t) A\left(G_{i}(t)\right) z_{i}(t) \\
& +R_{i}(t) B\left(G_{i}(t)\right) u\left(\Omega_{m-i}(t)\right) \\
& +L_{i}(t)\left[z_{i}\left(\theta_{i}^{-1}(t)\right)-z_{i-1}(t)\right], 2 \leq i \leq m
\end{aligned}
$$

with the choices

$$
\begin{aligned}
& L_{i}(t)=-I_{n}-R_{i}(t) A\left(G_{i}(t)\right) \\
& \text { and } G_{i}(t)=\Omega_{m}^{-1}\left(\Omega_{m-i}(t)\right),
\end{aligned}
$$

we have

$$
|(x(t), \mathcal{E}(t))| \leq \mu_{1}\left(|(x, \mathcal{E})|_{\left[-c_{h}, 0\right]} e^{-\mu_{2} t}+|\delta|_{\infty}\right)
$$

for all $t \geq 0$, where $\mathcal{E}(t)=\left(z_{1}(t)-x\left(\theta_{1}(t)\right), z_{2}(t)-\right.$ $\left.z_{1}\left(\theta_{2}(t)\right), \ldots, z_{m}(t)-z_{m-1}\left(\theta_{m}(t)\right)\right)$.

Remark 1 The $z_{i}$ dynamics in (10) are called the ith sequential subpredictor for each $i$. By the formula

$$
e^{u_{c}}=\lim _{m \rightarrow \infty}\left(\left(1+\frac{u_{c}}{m}\right)^{m / u_{c}}\right)^{u_{c}},
$$

condition (8) holds if $m$ is large enough. The estimate (12) says that the combined $(x, \mathcal{E})$ dynamics are exponentially input-to-state stable (or ISS) with respect to $\delta$; see Khalil (2002) for ISS properties. Three key features of the preceding construction are (a) our allowing arbitrarily large delay bounds $c_{h}>0$, (b) the fact that our control (9) has no distributed terms and no Lie derivatives, and that (c) only the bottom $n$ components of (10) are used to compute the control. This differs from standard predictive controls that contain distributed terms, and emulation controls that limit the allowable upper bounds on the delay. Longer delays and larger values of $|A|_{\infty}$ lead to larger $m$ values. See our examples below.

Remark 2 A motivation for (9)-(10) is that the maximum delay is divided among the predictors, and each predictor refers to a specific and constant time ahead. The $C^{1}$ property of $h$ is needed to ensure the $C^{1}$ properties of the $\theta_{i}$ 's in our stabilization analysis. Using Gaussian smoothing and interpolation, we can sometimes approximate discontinuous delays (which violate Assumption 1) by delays $h(t)$ that satisfy Assumption 1, and then we can use $h(t)$ in our analysis, so our Assumption 1 is widely applicable through delay approximations. However, 
one cannot extend our result to arbitrary sawtooth shaped delays (which arise from sampling), as illustrated by

$$
\begin{aligned}
& \dot{x}_{1}(t)=x_{2}(t) \\
& \dot{x}_{2}(t)=-x_{1}(t)+u\left(t_{k}\right), t \in\left[t_{k}, t_{k+1}\right)
\end{aligned}
$$

with the sample times $t_{k}=2 \pi k$ for all integers $k \geq 0$, where the control values $u\left(t_{k}\right)$ are arbitrary, since (14) cannot be globally asymptotically stabilized to 0 by any feedback. To see why (14) cannot be globally asymptotically stabilized to 0 , first notice that the function

$$
\gamma(t)=\cos (t) x_{1}(t)-\sin (t) x_{2}(t)
$$

satisfies $\dot{\gamma}(t)=-\sin (t) u\left(t_{k}\right)$ along all solutions of (14) for all $t \in\left[t_{k}, t_{k+1}\right)$ and integers $k \geq 0$, which gives

$$
\gamma(2 \pi(k+1))-\gamma(2 \pi k)=\int_{2 \pi k}^{2 \pi(k+1)} \dot{\gamma}(s) \mathrm{d} s=0
$$

so (15) gives $x_{1}(2 \pi(k+1))=\gamma(2 \pi(k+1))=\gamma(2 \pi k)=$ $\ldots=\gamma(0)=x_{1}(0)$ for all integers $k \geq 0$, so $x_{1}(t)$ cannot converge to 0 .

\section{Proof of Theorem 1}

We use this lemma, which we prove in the appendix:

Lemma 1 The $\theta_{i}$ 's in (3) and the constant (6) satisfy

$$
\left|\dot{\theta}_{i}(t)-1\right| \leq \frac{u_{c}}{m} \quad \text { and } \quad\left|\theta_{i}^{-1}(t)-t\right| \leq \frac{c_{h}}{1-l_{h}} \frac{1}{m}
$$

for all $t \geq 0$ and $i \in\{1,2, \ldots, m\}$.

By Lemma 1 and our definition of the $R_{i}$ 's in (4), we get

$$
\left|R_{i}(t)\right| \leq\left(1+\frac{u_{c}}{m}\right)^{m}, \quad 1 \leq i \leq m
$$

where throughout the proof, all equalities and inequalities are for all $t \geq 0$, unless otherwise indicated. In terms of the states $z_{i}$ of $(10)$, set $\xi_{i}(t)=z_{i-1}\left(\theta_{i}(t)\right)$ for $i \geq 1$, where $z_{0}=x$. The rest of the proof has four parts.

Part 1: Error Dynamics. We first derive a useful formula for the dynamics of the error $\mathcal{E}=\left(\mathcal{E}_{1}, \ldots, \mathcal{E}_{m}\right)$, where

$$
\mathcal{E}_{i}(t)=z_{i}(t)-\xi_{i}(t)=z_{i}(t)-z_{i-1}\left(\theta_{i}(t)\right)
$$

for all $i \in\{1, \ldots, m\}$. Our formulas (3) give

$$
\begin{aligned}
\dot{\xi}_{1}(t)= & \dot{\theta}_{1}(t) A\left(\theta_{1}(t)\right) \xi_{1}(t)+\dot{\theta}_{1}(t) \delta\left(\theta_{1}(t)\right) \\
& +\dot{\theta}_{1}(t) B\left(\theta_{1}(t)\right) u\left(\theta_{1}(t)-h\left(\theta_{1}(t)\right)\right) \\
= & R_{1}(t) A\left(\theta_{1}(t)\right) \xi_{1}(t)+\dot{\theta}_{1}(t) \delta\left(\theta_{1}(t)\right) \\
& +R_{1}(t) B\left(\theta_{1}(t)\right) u\left(\Omega_{m}\left(\theta_{1}(t)\right)\right),
\end{aligned}
$$

because

$$
\theta_{i}^{-1}(t) \leq t \text { for all } t \geq 0
$$

since (21) is equivalent to $\Omega_{m-i+1}(t) \leq \Omega_{m-i}(t)$, which is equivalent to $m-i+1 \geq m-i$, so $\theta_{1}(t) \geq t$. Since $\Omega_{m}\left(\theta_{1}(t)\right)=\Omega_{m-1}(t)$, we then have

$$
\begin{aligned}
& \dot{\xi}_{1}(t)=R_{1}(t) A\left(\theta_{1}(t)\right) \xi_{1}(t) \\
& +R_{1}(t) B\left(\theta_{1}(t)\right) u\left(\Omega_{m-1}(t)\right)+\dot{\theta}_{1}(t) \delta\left(\theta_{1}(t)\right) .
\end{aligned}
$$

Hence, (19) and the $z_{1}$ dynamics in (10) give

$$
\begin{aligned}
\dot{\mathcal{E}}_{1}(t)= & R_{1}(t) A\left(\theta_{1}(t)\right) \mathcal{E}_{1}(t)+L_{1}(t) \mathcal{E}_{1}\left(\theta_{1}^{-1}(t)\right) \\
& -\dot{\theta}_{1}(t) \delta\left(\theta_{1}(t)\right) .
\end{aligned}
$$

If $2 \leq i \leq m$, then we have $G_{i-1} \circ \theta_{i}=\Omega_{m}^{-1} \circ \Omega_{m-i+1} \circ$ $\Omega_{m-i+1}^{-1} \circ \Omega_{m-i}=G_{i}$, where $\circ$ denotes composition, so

$$
\begin{aligned}
\dot{\xi}_{i}(t)= & \dot{\theta}_{i}(t)\left\{R _ { i - 1 } ( \theta _ { i } ( t ) ) \left[A\left(G_{i}(t)\right) z_{i-1}\left(\theta_{i}(t)\right)\right.\right. \\
& \left.+B\left(G_{i}(t)\right) u\left(\Omega_{m-i+1}\left(\theta_{i}(t)\right)\right)\right] \\
& +L_{i-1}\left(\theta_{i}(t)\right)\left[z_{i-1}\left(\theta_{i-1}^{-1}\left(\theta_{i}(t)\right)\right)\right. \\
& \left.\left.-z_{i-2}\left(\theta_{i}(t)\right)\right]\right\} \\
= & R_{i}(t) B\left(G_{i}(t)\right) u\left(\Omega_{m-i+1}\left(\theta_{i}(t)\right)\right) \\
& +\dot{\theta}_{i}(t) L_{i-1}\left(\theta_{i}(t)\right)\left[z_{i-1}\left(\theta_{i-1}^{-1}\left(\theta_{i}(t)\right)\right)\right. \\
& \left.-z_{i-2}\left(\theta_{i}(t)\right)\right]+R_{i}(t) A\left(G_{i}(t)\right) \xi_{i}(t),
\end{aligned}
$$

by our definition of the $R_{i}$ 's. Since $\Omega_{m-i+1}\left(\theta_{i}(t)\right)=$ $\Omega_{m-i}(t)$, we deduce that for all $i \in\{2, \ldots, m\}$, we get

$$
\begin{aligned}
\dot{\mathcal{E}}_{i}(t)= & L_{i}(t)\left[z_{i}\left(\theta_{i}^{-1}(t)\right)-z_{i-1}(t)\right] \\
& -\dot{\theta}_{i}(t) L_{i-1}\left(\theta_{i}(t)\right)\left[z_{i-1}\left(\theta_{i-1}^{-1}\left(\theta_{i}(t)\right)\right)\right. \\
& \left.-z_{i-2}\left(\theta_{i}(t)\right)\right]+R_{i}(t) A\left(G_{i}(t)\right) \mathcal{E}_{i}(t) \\
= & R_{i}(t) A\left(G_{i}(t)\right) \mathcal{E}_{i}(t)+L_{i}(t) \mathcal{E}_{i}\left(\theta_{i}^{-1}(t)\right) \\
& -\dot{\theta}_{i}(t) L_{i-1}\left(\theta_{i}(t)\right) \mathcal{E}_{i-1}\left(\theta_{i-1}^{-1}\left(\theta_{i}(t)\right)\right) .
\end{aligned}
$$

Since $\theta_{1}=G_{1}$, this gives these error dynamics:

$$
\left\{\begin{aligned}
\dot{\mathcal{E}}_{1}(t)= & R_{1}(t) A\left(G_{1}(t)\right) \mathcal{E}_{1}(t) \\
& +L_{1}(t) \mathcal{E}_{1}\left(\theta_{1}^{-1}(t)\right)-\dot{\theta}_{1}(t) \delta\left(\theta_{1}(t)\right) \\
\dot{\mathcal{E}}_{i}(t)= & R_{i}(t) A\left(G_{i}(t)\right) \mathcal{E}_{i}(t)+L_{i}(t) \mathcal{E}_{i}\left(\theta_{i}^{-1}(t)\right) \\
& -\dot{\theta}_{i}(t) L_{i-1}\left(\theta_{i}(t)\right) \\
& \times \mathcal{E}_{i-1}\left(\theta_{i-1}^{-1}\left(\theta_{i}(t)\right)\right), 2 \leq i \leq m
\end{aligned}\right.
$$

We require the following preliminary analysis.

Part 2: Fundamental System. We first study the system

$$
\begin{aligned}
& \dot{s}_{i}(t)=R_{i}(t) A\left(G_{i}(t)\right) s_{i}(t)+L_{i}(t) s_{i}\left(\theta_{i}^{-1}(t)\right) \\
& =-s_{i}(t)-L_{i}(t)\left[s_{i}(t)-s_{i}\left(\theta_{i}^{-1}(t)\right)\right]
\end{aligned}
$$


for a fixed $i \in\{1,2, \ldots, m\}$, where the second equality used our choices $L_{i}(t)=-I_{n}-R_{i}(t) A\left(G_{i}(t)\right)$. By $(21)$, we obtain

$$
\begin{aligned}
& \dot{s}_{i}(t)=-s_{i}(t)-L_{i}(t) \int_{\theta_{i}^{-1}(t)}^{t} R_{i}(l) A\left(G_{i}(l)\right) s_{i}(l) \mathrm{d} l \\
& +L_{i}(t) \int_{\theta_{i}^{-1}(t)}^{t}\left(I_{n}+R_{i}(l) A\left(G_{i}(l)\right)\right) s_{i}\left(\theta_{i}^{-1}(l)\right) \mathrm{d} l .
\end{aligned}
$$

To study stability properties for $(28)$, we use $Q\left(s_{i}\right)=$ $\left|s_{i}\right|^{2} / 2$, and the lower bound $\theta_{i}^{-1}(t) \geq t-c_{h} /\left(\left(1-l_{h}\right) m\right)$, which follows from the second bound in (17).

By our choices (7) of $b_{1}$ and $b_{2}$, our second bound in (17) from Lemma 1, and (18), it follows that the time derivative of $Q$ along the trajectories of (28) satisfies

$$
\begin{aligned}
\dot{Q}(t) \leq & -\left|s_{i}(t)\right|^{2}+b_{1}\left|s_{i}(t)\right| \int_{t-\frac{c_{h}}{1-l_{h}} \frac{1}{m}}^{t}\left|s_{i}(l)\right| \mathrm{d} l \\
& +b_{2}\left|s_{i}(t)\right| \int_{\theta_{i}^{-1}\left(t-\frac{c_{h}}{1-l_{h}} \frac{1}{m}\right)}^{\theta_{i}^{-1}(t)}\left|s_{i}(r)\right|\left|\dot{\theta}_{i}(r)\right| \mathrm{d} r
\end{aligned}
$$

by using the change the variables $r=\theta_{i}^{-1}(l)$. Here and in what follows, all inequalities and equalities are for all $t \geq 2 c_{h} /\left(m\left(1-l_{h}\right)\right)$, unless otherwise indicated. Hence, since (21) is satisfied, the bounds (17) give

$$
\begin{aligned}
\dot{Q}(t) \leq & -\left|s_{i}(t)\right|^{2}+b_{1}\left|s_{i}(t)\right| \int_{t-\frac{c_{h}}{1-l_{h}} \frac{1}{m}}^{t}\left|s_{i}(l)\right| \mathrm{d} l \\
& +b_{3}\left|s_{i}(t)\right| \int_{t-\frac{2 c_{h}}{1-l_{h}} \frac{1}{m}}^{t}\left|s_{i}(r)\right| \mathrm{d} r .
\end{aligned}
$$

We can apply Young's inequality $s_{i}(t) r \leq \frac{1}{4}\left|s_{i}(t)\right|^{2}+r^{2}$ twice for suitable $r \geq 0$, and then Jensen's inequality (applied to the convex integrand $\left|s_{i}\right|^{2}$ ), to get

$$
\begin{aligned}
\dot{Q}(t) \leq & -\frac{1}{2}\left|s_{i}(t)\right|^{2}+\frac{b_{1}^{2} c_{h}}{\left(1-l_{h}\right) m} \int_{t-\frac{c_{h}}{1-l_{h}} \frac{1}{m}}^{t}\left|s_{i}(l)\right|^{2} \mathrm{~d} l \\
& +\frac{2 b_{3}^{2} c_{h}}{\left(1-l_{h}\right) m} \int_{t-\frac{2 c_{h}}{1-l_{h}} \frac{1}{m}}^{t}\left|s_{i}(r)\right|^{2} \mathrm{~d} r
\end{aligned}
$$

where the first application was

$$
\begin{aligned}
& b_{1}\left|s_{i}(t)\right| \int_{t-\frac{c_{h}}{\left(1-l_{h}\right) m}}^{t}\left|s_{i}(l)\right| \mathrm{d} l \\
& \leq \frac{1}{4}\left|s_{i}(t)\right|^{2}+\frac{b_{1}^{2} c_{h}}{\left(1-l_{h}\right) m} \int_{t-\frac{c_{h}}{\left(1-l_{h}\right) m}}^{t}\left|s_{i}(l)\right|^{2} \mathrm{~d} l,
\end{aligned}
$$

and the second one was analogous, except with $b_{1}$ and $c_{h}$ in (32) replaced by $b_{3}$ and $2 c_{h}$, respectively.
Since our condition (8) allows us to find a constant $\lambda>1$ that is close enough to 1 so that

$$
\lambda\left(\frac{c_{h}}{\left(1-l_{h}\right) m}\right)^{2}\left(b_{1}^{2}+4 b_{3}^{2}\right)<\frac{1}{2}
$$

it follows from (31) that we can also find a constant $c_{0}>0$ such that the time derivative of

$$
\begin{aligned}
& Q^{\sharp}\left(s_{i, t}\right)=Q\left(s_{i}(t)\right) \\
& +\frac{c_{h} \lambda}{\left(1-l_{h}\right) m}\left(b_{1}^{2} \int_{t-\frac{c_{h}}{1-l_{h}} \frac{1}{m}}^{t} \int_{w}^{t}\left|s_{i}(r)\right|^{2} \mathrm{~d} r \mathrm{~d} w\right. \\
& \left.+2 b_{3}^{2} \int_{t-\frac{2 c_{h}}{1-l_{h}} \frac{1}{m}}^{t} \int_{w}^{t}\left|s_{i}(r)\right|^{2} \mathrm{~d} r \mathrm{~d} w\right)
\end{aligned}
$$

along all solutions of (28) satisfies

$$
\dot{Q}^{\sharp} \leq-c_{0} Q^{\sharp}\left(s_{i, t}\right),
$$

where (34) was obtained using the relations

$$
\begin{aligned}
& \int_{t-\frac{j c_{h}}{1-l_{h}} \frac{1}{m}}^{t} \int_{w}^{t}\left|s_{i}(r)\right|^{2} \mathrm{~d} r \mathrm{~d} w \\
& \leq \frac{j c_{h}}{\left(1-l_{h}\right) m} \int_{t-\frac{j c_{h}}{1-l_{h}} \frac{1}{m}}^{t}\left|s_{i}(r)\right|^{2} \mathrm{~d} r
\end{aligned}
$$

and

$$
\begin{aligned}
& \frac{d}{d t}\left(\int_{t-\frac{j c_{h}}{1-l_{h}} \frac{1}{m}}^{t} \int_{w}^{t}\left|s_{i}(r)\right|^{2} \mathrm{~d} r \mathrm{~d} w\right) \\
& =\frac{j c_{h}}{\left(1-l_{h}\right) m}\left|s_{i}(t)\right|^{2}-\int_{t-\frac{j c_{h}}{\left(1-l_{h}\right) m}}^{t}\left|s_{i}(r)\right|^{2} \mathrm{~d} r
\end{aligned}
$$

for $j=1$ and $j=2$ and where $s_{i, t}(\ell)=s_{i}(t+\ell)$ for all $\ell \in\left[-2 c_{h} /\left(\left(1-l_{h}\right) m\right), 0\right]$. For instance, we can choose

$$
\begin{aligned}
& c_{0}= \\
& \min \left\{1-2 \lambda\left(\frac{c_{h}}{\left(1-l_{h}\right) m}\right)^{2}\left(b_{1}^{2}+4 b_{3}^{2}\right), \frac{(\lambda-1)\left(1-l_{h}\right) m}{2 \lambda c_{h}}\right\} .
\end{aligned}
$$

This concludes our analysis of (27).

Part 3: Input-to-State Stability of the Error Dynamics (26). We first study the dynamics for

$$
\mathcal{E}^{\sharp}(t)=\left(\mathcal{E}_{1}\left(\theta_{1}^{-1} \circ \theta_{m}(t)\right), \mathcal{E}_{2}\left(\theta_{2}^{-1} \circ \theta_{m}(t)\right), \ldots, \mathcal{E}_{m}(t)\right)
$$

with the disturbance $\delta\left(\theta_{m}(t)\right)$. We can find positive constants $\underline{\theta}$ and $\bar{\theta}$ such that $\underline{\theta} \leq\left(\theta_{i}^{-1} \circ \theta_{m}\right)^{\prime}(t) \leq \bar{\theta}$ hold for all $t \geq 0$ and $i \in\{1,2, \ldots, m\}$. To find $\underline{\theta}$ and $\bar{\theta}$, first note 
that for each $i \in\{1,2, \ldots, m\}$, we have

$$
\begin{aligned}
\theta_{i}^{-1} \circ \theta_{m} & =\left(\Omega_{m-i+1}^{-1} \circ \Omega_{m-i}\right)^{-1} \circ \Omega_{1}^{-1} \\
& =\Omega_{m-i}^{-1} \circ \Omega_{m-i+1} \circ \Omega_{1}^{-1},
\end{aligned}
$$

so the chain rule gives

$$
\begin{aligned}
& \left(\theta_{i}^{-1} \circ \theta_{m}\right)^{\prime}(t)=\left(1-\frac{m-i}{m} \dot{h}\left(\theta_{i}^{-1} \circ \theta_{m}(t)\right)\right)^{-1} \\
& \times\left(1-\frac{m-i+1}{m} \dot{h}\left(\Omega_{1}^{-1}(t)\right)\right) \\
& \times\left(1-\frac{1}{m} \dot{h}\left(\Omega_{1}^{-1}(t)\right)\right)^{-1}
\end{aligned}
$$

so the existence of the constants $\underline{\theta}$ and $\bar{\theta}$ follows from the lower and upper bounds on $\dot{h}$ from Assumption 1.

Hence, the decay estimate (34) along trajectories of (27), combined with Young's inequality and our choice of the quadratic part of $Q^{\sharp}$, implies that along all solutions of the dynamics for

$$
\left(\mathcal{E}_{1}^{\sharp}(t), \mathcal{E}_{2}^{\sharp}(t)\right)=\left(\mathcal{E}_{1}\left(\theta_{1}^{-1} \circ \theta_{m}(t)\right), \mathcal{E}_{2}\left(\theta_{2}^{-1} \circ \theta_{m}(t)\right)\right),
$$

we have

$$
\begin{aligned}
\frac{d}{d t} Q^{\sharp}\left(\mathcal{E}_{2, t}^{\sharp}\right) \leq & -\frac{\theta}{2} c_{0} Q^{\sharp}\left(\mathcal{E}_{2, t}^{\sharp}\right) \\
& +\frac{2 \bar{\theta}^{4}}{\underline{\theta} c_{0}}\left|\dot{\theta}_{2}\right|_{\infty}^{2}\left|L_{1}\right|_{\infty}^{2} Q^{\sharp}\left(\mathcal{E}_{1, t}^{\sharp}\right) \\
\frac{d}{d t} Q^{\sharp}\left(\mathcal{E}_{1, t}^{\sharp}\right) \leq & -\frac{\underline{\theta} c_{0}}{2} Q^{\sharp}\left(\mathcal{E}_{1, t}^{\sharp}\right)+\frac{\bar{\theta}^{4}}{\underline{\theta} c_{0}}\left|\dot{\theta}_{1}\right|_{\infty}^{2}|\delta|_{\infty}^{2},
\end{aligned}
$$

where the first inequality used Young's inequality to get

$$
\begin{aligned}
& \bar{\theta}^{2}\left|\mathcal{E}_{2}\left(\theta_{2}^{-1} \circ \theta_{m}(t)\right)\right|\left|\dot{\theta}_{2}\right|_{\infty}\left|L_{1}\right|_{\infty}\left|\mathcal{E}_{1}\left(\theta_{1}^{-1} \circ \theta_{m}(t)\right)\right| \\
& \leq \frac{\hat{\theta} c_{0}}{2} Q^{\sharp}\left(\mathcal{E}_{2, t}^{\sharp}\right)+\frac{2 \bar{\theta}^{4}}{\underline{\theta} c_{0}}\left(\left|\dot{\theta}_{2}\right|_{\infty}\left|L_{1}\right|_{\infty}\right)^{2} Q^{\sharp}\left(\mathcal{E}_{1, t}^{\sharp}\right),
\end{aligned}
$$

and the second inequality in (35) was obtained in an analogous way. Hence, the function

$$
\begin{aligned}
& Q_{2}^{\sharp}\left(\mathcal{E}_{1, t}^{\sharp}, \mathcal{E}_{2, t}^{\sharp}\right)= \\
& Q^{\sharp}\left(\mathcal{E}_{2, t}^{\sharp}\right)+\left(1+\left(\frac{2 \bar{\theta}^{2}\left|\dot{\theta}_{2}\right|_{\infty}\left|L_{1}\right|_{\infty}}{\underline{\theta} c_{0}}\right)^{2}\right) Q^{\sharp}\left(\mathcal{E}_{1, t}^{\sharp}\right)
\end{aligned}
$$

satisfies

$$
\begin{aligned}
\dot{Q}_{2}^{\sharp} \leq & -\frac{\underline{\theta} c_{0}}{2}\left(Q^{\sharp}\left(\mathcal{E}_{1, t}^{\sharp}\right)+Q^{\sharp}\left(\mathcal{E}_{2, t}^{\sharp}\right)\right) \\
& +\left(1+\left(\frac{2 \bar{\theta}^{2}\left|\dot{\theta}_{2}\right|_{\infty}\left|L_{1}\right|_{\infty}}{\underline{\theta} c_{0}}\right)^{2}\right) \frac{\bar{\theta}^{4}}{\underline{\theta} c_{0}}\left|\dot{\theta}_{1}\right|^{2}|\delta|_{\infty}^{2}
\end{aligned}
$$

and so is an ISS Lyapunov-Krasovskii functional for the $\left(\mathcal{E}_{1}^{\sharp}(t), \mathcal{E}_{2}^{\sharp}(t)\right)$ dynamics. Continuing in a similar way (by adding one component of $\mathcal{E}^{\sharp}$ at a time) provides positive constants $\omega_{i}$ and $\eta_{i}$ such that

$$
\begin{aligned}
& Q_{m}^{\sharp}\left(\mathcal{E}_{t}^{\sharp}\right)= \\
& \omega_{1} Q^{\sharp}\left(\mathcal{E}_{1, t}^{\sharp}\right)+\ldots+\omega_{m-1} Q^{\sharp}\left(\mathcal{E}_{m-1, t}^{\sharp}\right)+\omega_{m} Q^{\sharp}\left(\mathcal{E}_{m, t}^{\sharp}\right)
\end{aligned}
$$

satisfies

$$
\dot{Q}_{m}^{\sharp}\left(\mathcal{E}_{t}^{\sharp}\right) \leq-\eta_{1} Q_{m}^{\sharp}\left(\mathcal{E}_{t}^{\sharp}\right)+\eta_{2}|\delta|_{\infty}^{2}
$$

along all solutions of the $\mathcal{E}^{\sharp}$ dynamics.

Part 4: ISS of (1) with Our Control (9). By (19), we have

$$
\begin{aligned}
& z_{m}(t)=\xi_{m}(t)+\mathcal{E}_{m}(t)=z_{m-1}\left(\theta_{m}(t)\right)+\mathcal{E}_{m}(t) \\
& =z_{m-2}\left(\theta_{m-1}\left(\theta_{m}(t)\right)\right)+\mathcal{E}_{m-1}\left(\theta_{m}(t)\right)+\mathcal{E}_{m}(t)
\end{aligned}
$$

and therefore we can argue inductively to get

$$
\begin{aligned}
& z_{m}(t)=x\left(\theta_{1}\left(\ldots \theta_{m-1}\left(\theta_{m}(t)\right) \ldots\right)\right) \\
& +\mathcal{E}_{1}\left(\theta_{2}\left(\ldots \theta_{m}(t) \ldots\right)\right)+\ldots+\mathcal{E}_{m}(t) .
\end{aligned}
$$

The formulas $\theta_{i}(t)=\Omega_{m-i+1}^{-1}\left(\Omega_{m-i}(t)\right)$ then give $\theta_{m-1}\left(\theta_{m}(t)\right)=\Omega_{2}^{-1}\left(\Omega_{1}\left(\Omega_{1}^{-1}\left(\Omega_{0}(t)\right)\right)\right)=\Omega_{2}^{-1}(t)$. We deduce that $\theta_{1}\left(\ldots \theta_{m-1}\left(\theta_{m}(t)\right) \ldots\right)=\Omega_{m}^{-1}(t)$, and so $z_{m}(t)=x\left(\Omega_{m}^{-1}(t)\right)+\mathcal{E}_{1}\left(\theta_{2}\left(\ldots \theta_{m}(t) \ldots\right)\right)+\ldots+\mathcal{E}_{m}(t)$. Hence, our control (9) can be written as

$$
\begin{aligned}
& u(t-h(t))=K(t)\left[x\left(\Omega_{m}^{-1}(t-h(t))\right)\right. \\
& \left.+\mathcal{E}_{1}\left(\theta_{2}\left(\ldots \theta_{m}(t-h(t) \ldots)\right)\right)+\ldots+\mathcal{E}_{m}(t-h(t))\right] .
\end{aligned}
$$

Since $\Omega_{m}(t)=t-h(t)$, the closed loop system is then

$$
\begin{aligned}
& \dot{x}(t)=(A(t)+B(t) K(t)) x(t) \\
& +\left\{B ( t ) \left[K(t) \mathcal{E}_{1}\left(\theta_{2}\left(\ldots \theta_{m}(t-h(t) \ldots)\right)\right)\right.\right. \\
& \left.\left.+\ldots+K(t) \mathcal{E}_{m}(t-h(t))\right]+\delta(t)\right\}
\end{aligned}
$$

Also, Assumption 2 provides a $C^{1}$ bounded function $P$ such that the Lyapunov function $V(t, x)=x^{\top} P(t) x$ has a quadratic lower bound in $|x|$ and satisfies $\dot{V} \leq-|x(t)|^{2}$ along all solutions of (2) (using (Khalil, 2002, Theorem $4.14)$ ). Then the triangle inequality gives

$$
\begin{aligned}
\dot{V} \leq & -\frac{1}{2}|x(t)|^{2}+2|P|_{\infty}^{2}\left|\delta^{\sharp}\right|_{\infty}^{2} \\
\leq & -\frac{1}{2|P|_{\infty}} V(t, x(t))+2|P|_{\infty}^{2}\left|\delta^{\sharp}\right|_{\infty}^{2} \\
\leq & -\frac{1}{2|P|_{\infty}} V(t, x(t)) \\
& +2(m+1)\left(|P|_{\infty}|B|_{\infty}|K|_{\infty}\right)^{2} \\
& \times\left(\left|\mathcal{E}_{1}\left(\theta_{2}\left(\ldots \theta_{m}(t-h(t) \ldots)\right)\right)\right|^{2}+\ldots\right. \\
& \left.+\left|\mathcal{E}_{m}(t-h(t))\right|^{2}+|\delta|_{\infty}^{2}\right)
\end{aligned}
$$

along all solutions of (42), where $\delta^{\sharp}$ is the quantity in 
curly braces in (42) viewed as a sum of $m+1$ terms.

Also, for all $i \in\{1,2, \ldots, m-1\}$, we have

$$
\begin{aligned}
& \left|\mathcal{E}_{i}\left(\theta_{i+1} \circ \ldots \circ \theta_{m}(t-h(t))\right)\right|^{2} \\
& =2 Q\left(\mathcal{E}_{i}\left(\theta_{i+1} \circ \ldots \circ \theta_{m}(t-h(t))\right)\right) \\
& =2 Q\left(\mathcal{E}_{i}^{\sharp}\left(\mathcal{M}_{i}(t)\right)\right) \leq \frac{2}{\omega_{i}} Q_{m}^{\sharp}\left(\mathcal{E}_{\mathcal{M}_{i}(t)}^{\sharp}\right)
\end{aligned}
$$

where $\mathcal{M}_{i}(t)=\theta_{m}^{-1} \circ \theta_{i} \circ \theta_{i+1} \circ \ldots \circ \theta_{m}(t-h(t))=$ $\Omega_{1} \circ \Omega_{m-i+1}^{-1} \circ \Omega_{m}(t)$, and

$$
\left|\mathcal{E}_{m}(t-h(t))\right|^{2}=2 Q\left(\mathcal{E}_{m}(t-h(t))\right) \leq \frac{2}{\omega_{m}} Q_{m}^{\sharp}\left(\mathcal{E}_{\mathcal{M}_{m}(t)}^{\sharp}\right)
$$

where $\mathcal{M}_{m}(t)=t-h(t)=\Omega_{m}(t)$. We can find positive constants $\underline{\mathcal{M}}$ and $\overline{\mathcal{M}}$ such that $\underline{\mathcal{M}} \leq \dot{\mathcal{M}}_{i}(t) \leq \overline{\mathcal{M}}$ for all $t \geq 0$ and $i \in\{1,2, \ldots, m\}$, by reasoning as we did to obtain $\underline{\theta}$ and $\bar{\theta}$. This and (43) give the decay estimates

$$
\begin{aligned}
\dot{V} \leq & -\frac{1}{2|P|_{\infty}} V(t, x(t)) \\
& +2(m+1)\left(|P|_{\infty}|B|_{\infty}|K|_{\infty}\right)^{2} \\
& \times\left(\sum_{i=1}^{m} \frac{2}{\omega_{i}} Q_{m}^{\sharp}\left(\mathcal{E}_{\mathcal{M}_{i}(t)}^{\sharp}\right)+|\delta|_{\infty}^{2}\right)
\end{aligned}
$$

and

$$
\frac{d}{d t} Q_{m}^{\sharp}\left(\mathcal{E}_{\mathcal{M}_{i}(t)}^{\sharp}\right) \leq-\underline{\mathcal{M}} \eta_{1} Q_{m}^{\sharp}\left(\mathcal{E}_{\mathcal{M}_{i}(t)}^{\sharp}\right)+\overline{\mathcal{M}} \eta_{2}|\delta|_{\infty}^{2}
$$

which imply that

$$
\begin{aligned}
& Q_{+}^{\sharp}\left(\mathcal{E}_{t}^{\sharp}\right)=V(t, x(t)) \\
& +\left(1+\frac{2(m+1)}{\underline{\mathcal{M}} \eta_{1}}\left(|P|_{\infty}|B|_{\infty}|K|_{\infty}\right)^{2}\right) \\
& \quad \times \sum_{i=1}^{m} \frac{2}{\omega_{i}} Q_{m}^{\sharp}\left(\mathcal{E}_{\mathcal{M}_{i}(t)}^{\sharp}\right)
\end{aligned}
$$

admits constants $d_{0}>0$ and $d_{1}>0$ such that the ISS Lyapunov-Krasovskii decay condition

$$
\dot{Q}_{+}^{\sharp}\left(\mathcal{E}_{t}^{\sharp \sharp}\right) \leq-d_{0} Q_{+}^{\sharp}\left(\mathcal{E}_{t}^{\sharp \sharp}\right)+d_{1}|\delta|_{\infty}^{2}
$$

holds along all solutions of the dynamics for

$$
\mathcal{E}^{\sharp \sharp}(t)=\left(x(t), \mathcal{E}_{\mathcal{M}_{1}(t)}^{\sharp}, \ldots, \mathcal{E}_{\mathcal{M}_{m}(t)}^{\sharp}\right) .
$$

Also, for all $t \geq 0$ and $i \in\{1,2, \ldots, m\}$, we can use (21) to obtain $\theta_{i}^{-1} \circ \theta_{m} \circ \mathcal{M}_{i}(t)=\theta_{i}^{-1} \circ \Omega_{m-i+1}^{-1} \circ \Omega_{m}(t) \leq$ $\Omega_{m-i+1}^{-1} \circ \Omega_{m}(t) \leq t$. Hence, there are positive constants $c_{1}$ and $c_{2}$ such that along all solutions of the dynamics for $\mathcal{E}^{\sharp \sharp}$ and for all $i \in\{1,2, \ldots, m\}$, we have

$$
\begin{aligned}
& \left|\mathcal{E}_{i}\left(\theta_{i}^{-1} \circ \theta_{m} \circ \mathcal{M}_{i}(t)\right)\right|^{2} \\
& \leq Q_{+}^{\sharp}\left(\mathcal{E}_{t}^{\sharp \sharp}\right) \leq c_{1}\left(|(x, \mathcal{E})|_{\left[-c_{h}, 0\right]}^{2} e^{-c_{2} t}+|\delta|_{\infty}^{2}\right)
\end{aligned}
$$

along all solutions of the dynamics for (47) for all $t$ such that $\min _{i} \theta_{i}^{-1} \circ \theta_{m} \circ \mathcal{M}_{i}(t) \geq 0$. The final exponential ISS estimate for the $(x, \mathcal{E})$ dynamics can now be deduced from the quadratic structure of $V$ and $Q$, the invertibility of the functions $\mathcal{H}_{i}=\theta_{i}^{-1} \circ \theta_{m} \circ \mathcal{M}_{i}$ for all $i$, the existence of positive constants $h_{a}$ and $h_{b}$ such that $\mathcal{H}_{i}^{-1}(t) \geq h_{a} t-$ $h_{b}$ for all $i$ and $t \geq 0$ (which follows from our bounds on $\dot{h}$ from Assumption 1 and the Mean Value Theorem), and the subadditivity of the square root. This proves Theorem 1.

Remark 3 Our proof of Theorem 1 shows that our control can be written as

$$
\begin{aligned}
& u(t-h(t))=K(t) x(t)+ \\
& K(t)\left[\mathcal{E}_{1}\left(\theta_{2}\left(\ldots \theta_{m}(t-h(t) \ldots)\right)\right)+\right. \\
& \left.\ldots+\mathcal{E}_{m}(t-h(t))\right]
\end{aligned}
$$

which may at first seem to contradict our assumption that the current value of the state $x(t)$ is not available to use in the feedback control for (1). However, since

$$
\begin{aligned}
& \theta_{2} \circ \ldots \circ \theta_{m}(t-h(t)) \\
& =\Omega_{m-1}^{-1} \circ \Omega_{m-2} \circ \Omega_{m-2}^{-1} \circ \Omega_{m-3} \circ \ldots \circ \Omega_{1}^{-1} \circ \Omega_{m}(t) \\
& =\Omega_{m-1}^{-1} \circ \Omega_{m}(t)=\theta_{1}^{-1}(t)
\end{aligned}
$$

holds for all $t \geq 0$, we can use (19) to get

$$
\begin{aligned}
& \mathcal{E}_{1}\left(\theta_{2}\left(\ldots \theta_{m}(t-h(t) \ldots)\right)\right) \\
& =z_{1}\left(\theta_{1}^{-1}(t)\right)-\xi_{1}\left(\theta_{1}^{-1}(t)\right)=z_{1}\left(\theta_{1}^{-1}(t)\right)-x(t),
\end{aligned}
$$

so the two terms in (48) and (49) containing $x(t)$ cancel. Hence, our control (9) does not require current values $x(t)$.

Remark 4 Instead of (8), the main result in Mazenc and Malisoff (2016) required that $m$ satisfy

$$
\max \left\{1,4\left(\frac{b_{1}}{\sqrt{2}}+b_{3}\right) \frac{c_{h}}{1-l_{h}}\right\}<m
$$

where the $b_{i}$ are as defined in (7). The statement of the theorem in Mazenc and Malisoff (2016) is the same as in our theorem above for the special case where $\delta=0$, except for the condition on $m$. The sketch of the proof of the main result in Mazenc and Malisoff (2016) had the same structure as the complete proof we gave above with the same choice of $Q$, except that instead of (31), it used 
the estimates

$$
\begin{aligned}
\dot{Q}(t) \leq & -\frac{1}{2}\left|s_{i}(t)\right|^{2} \\
& +\frac{\left(b_{1} / \sqrt{2}\right)^{2}}{b_{1} / \sqrt{2}+b_{3}} \int_{t-\frac{c_{h}}{1-l_{h}} \frac{1}{m}}^{t}\left|s_{i}(l)\right|^{2} \mathrm{~d} l \\
& +\frac{b_{3}^{2}}{b_{1} / \sqrt{2}+b_{3}} \int_{t-\frac{2 c_{h}}{1-l_{h}} \frac{1}{m}}^{t}\left|s_{i}(r)\right|^{2} \mathrm{~d} r \\
\leq & -\frac{1}{2}\left|s_{i}(t)\right|^{2} \\
& +\left(\frac{b_{1}}{\sqrt{2}}+b_{3}\right) \int_{t-\frac{2 c_{h}}{1-l_{h}} \frac{1}{m}}^{t}\left|s_{i}(r)\right|^{2} \mathrm{~d} r
\end{aligned}
$$

which follow from Jensen's and Young's inequalities, using the fact that (50) implies that

$$
\frac{2 c_{h}}{m\left(1-l_{h}\right)}<\frac{1}{b_{1} / \sqrt{2}+b_{3}}
$$

as well as the estimate $\left(r^{2}+s^{2}\right) /(r+s) \leq r+s$, with the choices $r=b_{1} / \sqrt{2}$ and $s=b_{3}$. Then (50) implies that there are constants $c_{0}>0$ and $\lambda>1$ such that along all solutions of the fundamental system (27), the function

$$
\begin{aligned}
& Q_{\text {old }}^{\sharp}\left(s_{i, t}\right)=Q\left(s_{i}(t)\right) \\
& +\lambda\left(\frac{b_{1}}{\sqrt{2}}+b_{3}\right) \int_{t-\frac{2 c_{h}}{1-l_{h}} \frac{1}{m}}^{t} \int_{w}^{t}\left|s_{i}(r)\right|^{2} \mathrm{~d} r \mathrm{~d} w
\end{aligned}
$$

satisfies a decay estimate of the form (34), except with $Q^{\sharp}$ replaced by $Q_{\text {old }}^{\sharp}$. By replacing (50) by (8), we can allow smaller values $m$, which is useful in applications. In the next section, we compare the values of $m$ that can be obtained from (50) with the ones that we can obtain using our improved formula (8), in two examples.

\section{Examples}

Assumptions 1-2 hold for broad classes of systems of the form $\dot{x}(t)=A(t) x(t)+B(t) u(t-h(t))$, including all systems with constant controllable pairs $(A, B)$. We first illustrate Theorem 1 in a scalar case. Then we revisit the linearized pendulum from Mazenc et al. (2014). Our work Mazenc et al. (2014) covered the linearized pendulum under constant delays, using a predictive controller with distributed terms. Hence, this section improves on Mazenc and Malisoff (2017) and Mazenc et al. (2014) in the case of the pendulum because in what follows, we allow nonconstant delays and because our control has no distributed terms. We also study a dynamics from identification theory with a time-varying delay, which we studied under constant delays in Mazenc et al. (2008).

\subsection{Scalar Example}

Consider the scalar case

$$
\dot{x}(t)=r x(t)+u(t-h(t))+\delta(t)
$$

for any constant $r>0$ with the oscillating delay

$$
h(t)=1+\alpha \sin (t)
$$

for any constant $\alpha \in(0,1)$. Then Assumptions 1-2 are satisfied using $n=1, A=r, B=1, K=-2 r, c_{h}=$ $1+\alpha$, and $n_{h}=l_{h}=\alpha$, so our ISS conclusion from Theorem 1 is satisfied using the control $u(t)=-2 r z_{m}(t)$, where $z_{m}$ is the $m$ th component of the dynamic extension from Theorem 1, under our conditions on $m$. To compute lower bounds on the allowable values of $m$, notice that in terms of our notation (6)-(7), we have

$$
\begin{aligned}
& b_{1}=r\left(1+r\left(1+\frac{u_{c}}{m}\right)^{m}\right)\left(1+\frac{u_{c}}{m}\right)^{m}, \\
& b_{2}=\left(1+r\left(1+\frac{u_{c}}{m}\right)^{m}\right)^{2}, u_{c}=\frac{2 \alpha}{(1-\alpha)^{2}}, \\
& \text { and } b_{3}=\left(1+r\left(1+\frac{u_{c}}{m}\right)^{m}\right)^{2}\left(1+\frac{u_{c}}{m}\right) .
\end{aligned}
$$

Hence, our requirement on $m \geq 2$ from (50) is

$$
\begin{aligned}
& m-4 \frac{1+\alpha}{1-\alpha}\left[\frac{r}{\sqrt{2}}\left(1+r\left(1+\frac{u_{c}}{m}\right)^{m}\right)\left(1+\frac{u_{c}}{m}\right)^{m}\right. \\
& \left.+\left(1+r\left(1+\frac{u_{c}}{m}\right)^{m}\right)^{2}\left(1+\frac{u_{c}}{m}\right)\right]>0 .
\end{aligned}
$$

For any constants $\alpha \in(0,1)$ and $r>0$, we can compute the left side of $(56)$ as a function $\phi_{\alpha, r}(m)$ of $m$ and choose $m$ such that $\phi_{\alpha, r}(m)>0$, since $\lim _{m \rightarrow+\infty} \phi_{\alpha, r}(m)=+\infty$. For instance, if $\alpha=1 / 7$ and $r=1 / 2$, then $\phi_{1 / 7,1 / 2}(22)=0.847755$, and 22 is the smallest integer $m$ for which (56) is satisfied for these $\alpha$ and $r$ values. On the other hand, our condition (8) holds with $m=12$ with the preceding parameter values for $\alpha$ and $r$, so Theorem 1 applies with $m=12$. Hence, our new condition (8) on $m$ produced a $45 \%$ reduction in the required number $m$ of sequential subpredictors, as compared with (50). If we change the $\alpha$ value to $\alpha=1 / 5$ and keep the other parameters the same, then the smallest values of $m$ for which (56) and (8) are satisfied are $m=31$ and $m=17$, respectively, so our new method for computing the number of required subpredictors again produces significant reductions in $m$. In general, increasing $\alpha$ can require increasing $m$, with our new method in this work producing smaller minimum allowable $m$ values. See Fig. 1 for a comparison between the method for finding $m$ from Mazenc and Malisoff (2016) with the method from Theorem 1 above for the preceding scalar dynamics.

\subsection{Pendulum}

Consider the model

$$
\left\{\begin{array}{l}
\dot{r}_{1}(t)=r_{2}(t) \\
\dot{r}_{2}(t)=-\frac{g}{l} \sin \left(r_{1}(t)\right)+\frac{1}{\mathcal{M} l^{2}} v(t-h(t))
\end{array}\right.
$$



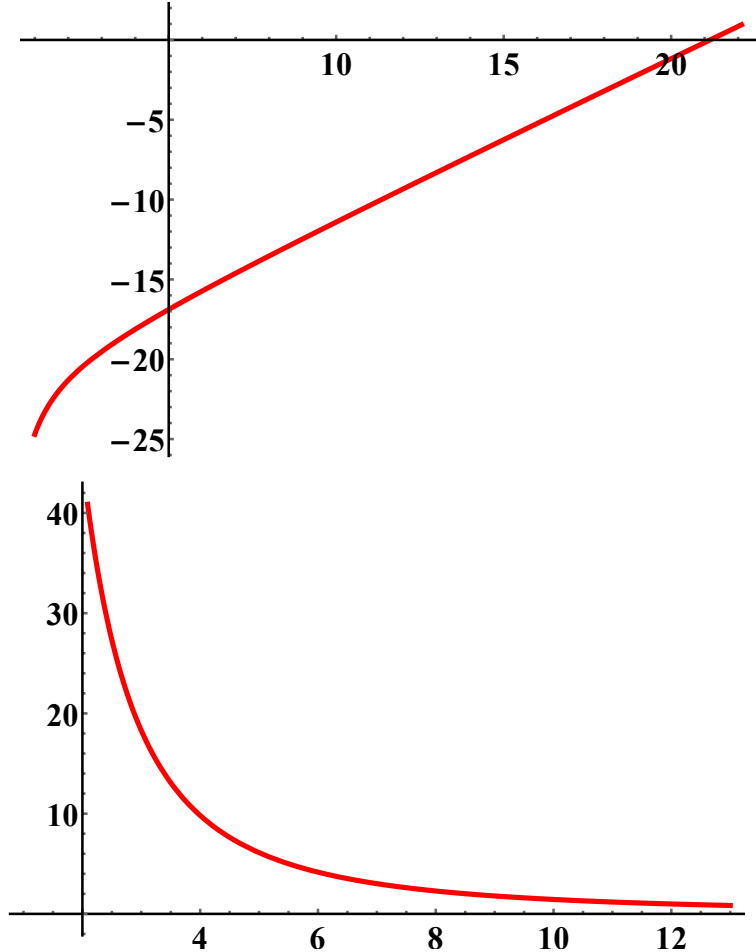

Fig. 1. Criteria for Choosing $m$ in Scalar Example. Top: $\phi_{1 / 7,1 / 2}(m)$ Showing Smallest Value $m=22$ for Which (56) is satisfied. Bottom: Left Side of (8) with $|A|_{\infty}=r=1 / 2$ Showing Smallest Value $m=12$ for which (8) Holds.

of the simple pendulum with a time-varying delay $h(t)$, where $g=9.8 \mathrm{~m} / \mathrm{s}$ is the gravitational constant, $l$ is the pendulum length in meters, $\mathcal{M}$ is the pendulum mass, and $v$ is the input. As in Mazenc and Malisoff (2017) and Mazenc et al. (2014), our control objective is to track a given $C^{1}$ reference trajectory $\left(r_{1, s}(t), r_{2, s}(t)\right)$ such that $\dot{r}_{1, s}(t)=r_{2, s}(t)$. Using the error variables $\tilde{r}_{i}=r_{i}-r_{i, s}(t)$ for $i=1,2$ and the change of feedback

$u(t-h(t))=\frac{1}{\mathcal{M} l^{2}} v(t-h(t))-\dot{r}_{2, s}(t)-\frac{g}{l} \sin \left(r_{1, s}(t)\right)$,

we obtain the tracking system

$$
\left\{\begin{aligned}
\dot{\tilde{r}}_{1}(t)= & \tilde{r}_{2}(t) \\
\dot{\tilde{r}}_{2}(t)= & \frac{g}{l}\left[\sin \left(r_{1, s}(t)\right)-\sin \left(\tilde{r}_{1}(t)+r_{1, s}(t)\right)\right] \\
& +u(t-h(t)) .
\end{aligned}\right.
$$

Our work in Mazenc et al. (2014) showed that when $r_{1, s}(t)=\omega t$ and $\omega>0$ is a large enough constant and the delay is the constant $h=1$, the linearization

$$
\left\{\begin{array}{l}
\dot{x}_{1}(t)=x_{2}(t) \\
\dot{x}_{2}(t)=-\frac{g}{l} \cos (\omega t) x_{1}(t)+u(t-h)
\end{array}\right.
$$

of (58) at 0 has the globally exponentially stabilizing distributed control $u$ defined by

$$
\begin{aligned}
u(t)= & -0.6 x_{1}(t)-0.4 x_{2}(t) \\
& -\int_{t-1}^{t}(0.6(t-r-1)+0.4) u(r) \mathrm{d} r .
\end{aligned}
$$

Our work Mazenc and Malisoff (2017) provided globally asymptotically stabilizing sequential predictor controls for the nonlinear system (58) when $h$ is constant. However, it is not obvious how to extend Mazenc and Malisoff (2017) to time-varying delays. Therefore, we apply Theorem 1 to its linearization (59) using the delay (54), where $\alpha \in(0,1)$ is a known constant, but analogous reasoning applies for any delay $h(t)$ satisfying Assumption 1.

To this end, first note that for any choice of the constant $\omega>0$, Assumption 2 is satisfied using

$$
\begin{aligned}
& A(t)=\left[\begin{array}{cc}
0 & 1 \\
-\frac{g}{l} \cos (\omega t) & 0
\end{array}\right], B=\left[\begin{array}{l}
0 \\
1
\end{array}\right], \\
& \text { and } K(t)=\left[\frac{g}{l} \cos (\omega t)-1 \quad-1\right]
\end{aligned}
$$

and condition (50) becomes

$$
\begin{aligned}
& \frac{m}{4}-\frac{1+\alpha}{1-\alpha}\left[\frac{\max \{g / l, 1\}}{\sqrt{2}}\left(1+\max \left\{\frac{g}{l}, 1\right\} u_{c, m}^{m}\right) u_{c, m}^{m}\right. \\
& \left.+\left(1+\max \left\{\frac{g}{l}, 1\right\} u_{c, m}^{m}\right)^{2} u_{c, m}\right] \\
& >0, \text { where } u_{c, m}=1+\frac{u_{c}}{m} .
\end{aligned}
$$

Condition (62) holds for any choices of $\alpha$ and $l$, by choosing $m$ large enough. For instance, if we pick $\alpha=1 / 7$, any $\omega>0$, any $l>g$, and $m=47$, then we get the value 0.376057 for the left side of (62), and 47 is the smallest integer $m$ such that (62) holds with the preceding parameter choices. On the other hand, (8) holds with $m=25$ with the preceding parameter choices, so Theorem 1 applies with $m=25$. Hence, we get a $47 \%$ reduction in the value of $m$ using our new condition (8). See Fig. 2 for a comparison between the two conditions (8) and (62) for choosing $m$ for the pendulum example. If we change $\alpha$ to $\alpha=1 / 5$ and keep the other parameters the same, then the conditions (8) and (62) for choosing $m$ give the minimum allowable values $m=38$ and $m=73$, respectively, so our new method again gives smaller values for the minimum required numbers $m$ of sequential predictors, and in general, larger $\alpha$ 's can require larger $m$ values.

\subsection{Identification Theory Example}

Theorem 1 applies to general time-varying linear systems for which the pairs $(A(t), B(t))$ are uniformly controllable and in particular allows nonzero drift, but in the special case where the drift is 0 , Theorem 1 gives an explicit lower bound on the allowable $m$ values. To 

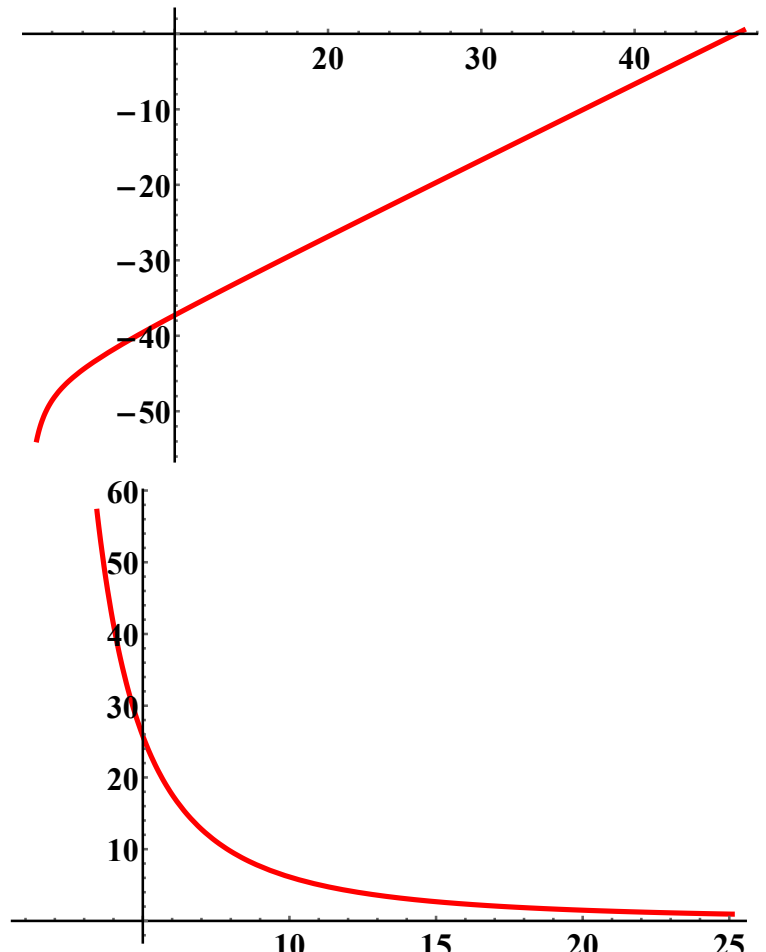

Fig. 2. Criteria for Choosing $m$ in Pendulum Example. Top: $\phi_{1 / 7,1}(m)$ Showing Smallest Value $m=47$ for Which (62) is satisfied. Bottom: Left Side of (8) with $|A|_{\infty}=1$ Showing Smallest Value $m=25$ for which (8) Holds.

illustrate this point, consider the system

$$
\dot{x}(t)=-\mathcal{N}(t) \mathcal{N}^{T}(t)[u(t-h(t))+\delta(t)]
$$

in which $\mathcal{N}: \mathbb{R} \rightarrow \mathbb{R}^{n}$ is a continuous function such that (i) $|\mathcal{N}(t)|=1$ for all $t \in \mathbb{R}$ and (ii) there are constants $\alpha^{\prime} \in(0,1), \beta^{\prime}>0$ and $\tilde{c}>0$ such that for all $t \in \mathbb{R}$, we have

$$
\alpha^{\prime} I_{n} \leq \int_{t}^{t+\tilde{c}} \mathcal{N}(\tau) \mathcal{N}^{T}(\tau) \mathrm{d} \tau \leq \beta^{\prime} I_{n},
$$

where $M_{1} \leq M_{2}$ for matrices $M_{1}$ and $M_{2}$ means that $M_{2}-M_{1}$ is positive semi-definite, and where the dimension $n$ of the state $x$ is arbitrary.

The system (63) is a benchmark model from identification theory, which we studied in Mazenc et al. (2008), using emulation methods. See (Anderson, 1977, Appendix A.1) for the derivation of (63) in a special case where the uncertainty $\delta=0$, where the dynamics are shown to arise when one wishes to identify coefficients of a stable plant transfer function, and see Aeyels and Sepulchre (1994), Aeyels et al. (1998), and Peuteman and Aeyels (2002) for more recent results on (63). However, the preceding papers do not cover time-varying input delays, so our sequential subpredictors work seems novel and significant, even in the special case of identification dynamics.
To apply our method to (63), first note that (Mazenc et al., 2008, Lemma 8) says that with the choices

$$
\begin{aligned}
& \kappa=1+\frac{\tilde{c}}{2}+\frac{1}{4 \alpha^{\prime}} \tilde{c}^{4} \text { and } \\
& P(t)=\kappa I_{n}+\int_{t-\tilde{c}}^{t} \int_{s}^{t} \mathcal{N}(l) \mathcal{N}^{T}(l) \mathrm{d} l \mathrm{~d} s,
\end{aligned}
$$

the function $V(x, t)=x^{T} P(t) x$ satisfies

$$
\dot{V} \leq-\alpha^{\prime}|x|^{2} / 2
$$

along all trajectories $\dot{x}(t)=-\mathcal{N}(t) \mathcal{N}^{T}(t) x(t)$, and $|P(t)| \leq \kappa+\tilde{c}^{2}$ for all $t \geq 0$, which implies that Assumption 2 is satisfied with $K=I_{n}$.

Hence, Theorem 1 applies for all choices of $h(t)$ that satisfy Assumption 1. Since (63) has the drift $A(t)=0$, our constant $b_{1}$ is zero and $b_{2}=1$, and our condition (8) on $m$ becomes

$$
2\left(\frac{c_{h}}{\left(1-l_{h}\right) m}\right)^{2} 4\left(1+\frac{u_{c}}{m}\right)^{2}<1
$$

By multiplying (67) through by $m^{4}$ and taking square roots of both sides and finally completing the square, we obtain the explicit requirement

$$
\sqrt{\left(\frac{\sqrt{2} c_{h}}{1-l_{h}}\right)^{2}+\frac{2 \sqrt{2} c_{h} u_{c}}{1-l_{h}}}+\frac{\sqrt{2} c_{h}}{1-l_{h}}<m
$$

on the number $m$ of sequential subpredictors for our conclusions of Theorem 1 to hold for our system (63).

\section{Conclusions and Future Work}

We designed exponentially ISS sequential predictor controllers for a large class of time-varying linear systems that satisfy a uniform controllability condition, under general conditions on the time-varying input delays. Our closed loop systems are ISS with respect to additive uncertainty, and our controls do not contain any distributed terms of the type that would otherwise arise when using reduction model controllers. By allowing arbitrarily large bounds on the input delays, we also overcame a possible challenge in applying emulation methods to systems that can have long input delays. Our strategy for compensating for large delay bounds involves increasing the numbers of sequential subpredictors, and our theorem provides a useful mechanism for computing the required number of subpredictors.

We hope to extend our work to adaptive systems, where additional dynamic extensions would make it possible to identify unknown parameters. We also hope to cover nonlinear systems with time-varying delays, by building sequential predictors for the linear parts, and then proving that the controls ensure local stabilization of the original nonlinear systems and using the Lyapunov 
decay conditions to estimate the region of attraction, which would be a sequential predictor analog of our work in Mazenc and Malisoff (2014) on local stabilization of nonlinear systems under reduction model controls. Finally, we plan to merge our methods with the methods in Malisoff and Zhang (2015) for state constrained curve tracking problems, using robust forward invariance.

\section{Appendix: Proof of Lemma 1}

Fix any $i \in\{1,2, \ldots, m\}$ and $t \geq 0$. By (3), we have $\theta_{i}(t)=\Omega_{m-i+1}^{-1}\left(\Omega_{m-i}(t)\right)$ and $\Omega_{m-i}(t)=\Omega_{m-i+1}(t)+$ $\frac{1}{m} h(t)$, so

$$
\begin{aligned}
\theta_{i}(t)= & t+\Omega_{m-i+1}^{-1}\left(\Omega_{m-i+1}(t)+\frac{1}{m} h(t)\right) \\
& -\Omega_{m-i+1}^{-1}\left(\Omega_{m-i+1}(t)\right)
\end{aligned}
$$

Hence, the Mean Value Theorem provides a $w \in \mathbb{R}$ (depending on $t$ and $i$ in general) such that

$$
\theta_{i}(t)=t+\frac{1}{\dot{\Omega}_{m-i+1}(w)} \frac{1}{m} h(t)
$$

Therefore, Assumption 1 gives

$$
\left|\theta_{i}(t)-t\right| \leq \frac{1}{1-l_{h}} \frac{1}{m} h(t) \leq \frac{c_{h}}{1-l_{h}} \frac{1}{m}
$$

Hence, the second inequality in (17) follows by replacing $t$ by $\theta_{i}^{-1}(t)$ in (A.3).

To check the first inequality in (17), first note that

$$
\begin{aligned}
\dot{\theta}_{i}(t)= & \frac{1-\frac{m-i}{m} \dot{h}(t)}{1-\frac{m-i+1}{m} \dot{h}\left(\Omega_{m-i+1}^{-1}\left(\Omega_{m-i}(t)\right)\right)} \\
= & \frac{1-\frac{m-i+1}{m} \dot{h}(t)}{1-\frac{m-i+1}{m} \dot{h}\left(\Omega_{m-i+1}^{-1}\left(\Omega_{m-i}(t)\right)\right)} \\
& +\frac{\frac{1}{m} \dot{h}(t)}{1-\frac{m-i+1}{m} \dot{h}\left(\Omega_{m-i+1}^{-1}\left(\Omega_{m-i}(t)\right)\right)} .
\end{aligned}
$$

Since $\Omega_{m-i}(t)=\Omega_{m-i+1}(t)+\frac{1}{m} h(t)$, the function

$$
\mathcal{G}_{i}(t)=\Omega_{m-i+1}^{-1}\left(\Omega_{m-i+1}(t)+\frac{1}{m} h(t)\right)
$$

is such that

$$
\begin{aligned}
\dot{\theta}_{i}(t)= & \frac{1-\frac{m-i+1}{m} \dot{h}(t)}{1-\frac{m-i+1}{m} \dot{h}\left(\mathcal{G}_{i}(t)\right)} \\
& +\frac{\frac{1}{m} \dot{h}(t)}{1-\frac{m-i+1}{m} \dot{h}\left(\Omega_{m-i+1}^{-1}\left(\Omega_{m-i}(t)\right)\right)} \\
= & \frac{m-i+1}{m} \frac{\dot{h}\left(t+\mathcal{G}_{i}(t)-\Omega_{m-i+1}^{-1}\left(\Omega_{m-i+1}(t)\right)\right)-\dot{h}(t)}{1-\frac{m-i+1}{m} \dot{h}\left(\mathcal{G}_{i}(t)\right)} \\
& +\frac{1}{m} \frac{\dot{h}(t)}{1-\frac{m-i+1}{m} \dot{h}\left(\Omega_{m-i+1}^{-1}\left(\Omega_{m-i}(t)\right)\right)}+1,
\end{aligned}
$$

by writing $\dot{h}(t)$ in the first numerator in (A.6) as $\dot{h}(t)=$

$$
\begin{aligned}
& \left(\dot{h}(t)-\dot{h}\left(\mathcal{G}_{i}(t)\right)+\dot{h}\left(\mathcal{G}_{i}(t)\right)\right. \text {. It follows that } \\
& \begin{aligned}
\left|\dot{\theta}_{i}(t)-1\right| \leq & \frac{m-i+1}{m} n_{h} \frac{\left|\mathcal{G}_{i}(t)-\Omega_{m-i+1}^{-1}\left(\Omega_{m-i+1}(t)\right)\right|}{1-\frac{m-i+1}{m} l_{h}} \\
& +\frac{1}{m} \frac{l_{h}}{1-\frac{m-i+1}{m} l_{h}},
\end{aligned}
\end{aligned}
$$

using the Lipschitz constant $n_{h}$ for $\dot{h}$. Hence, our choice of $\mathcal{G}_{i}$ and the Mean Value Theorem applied to $\Omega_{m-i+1}^{-1}$ give

$$
\begin{aligned}
\left|\dot{\theta}_{i}(t)-1\right| \leq & \frac{m-i+1}{m} n_{h} \frac{\left|\Omega_{m-i+1}(t)+\frac{1}{m} h(t)-\Omega_{m-i+1}(t)\right|}{\left(1-\frac{m-i+1}{m} l_{h}\right)^{2}} \\
& +\frac{1}{m} \frac{l_{h}}{1-\frac{m-i+1}{m} l_{h}} \\
\leq & \frac{1}{m} \frac{m-i+1}{m} n_{h} \frac{c_{h}}{\left(1-\frac{m-i+1}{m} l_{h}\right)^{2}}+\frac{1}{m} \frac{l_{h}}{1-\frac{m-i+1}{m} l_{h}} .
\end{aligned}
$$

The lemma now follows from our formula (6) for $u_{c}$.

\section{References}

Aeyels, D. and R. Sepulchre (1994). On the convergence of a time-variant linear differential equation arising in identification. Kybernetika 30, 715-723.

Aeyels, D., R. Sepulchre and J. Peuteman (1998). Asymptotic stability for time-variant systems and observability: uniform and nonuniform criteria. Mathematics of Control, Signals, and Systems 11, 1-27.

Ahmed-Ali, T., E. Cherrier and F. Lamnabhi-Lagarrigue (2012). Cascade high gain predictors for a class of nonlinear systems. IEEE Transactions on Automatic Control 57(1), 221-226.

Ahmed-Ali, T., I. Karafyllis and F. LamnabhiLagarrigue (2013). Global exponential sampled-data observers for nonlinear systems with delayed measurements. Systems and Control Letters 67(7), 539-549.

Ahmed-Ali, T., I. Karafyllis, M. Krstic and F. Lamnabhi-Lagarrigue (2016). Robust stabilization of nonlinear globally Lipschitz delay systems. Vol. 4 of Advances in Delays and Dynamics. Springer. New York. pp. $43-60$.

Anderson, B. (1977). Exponential stability of linear equations arising in adaptive identification. IEEE Transactions on Automatic Control 22(1), 83-88.

Artstein, Z. (1982). Linear systems with delayed controls: a reduction. IEEE Transactions on Automatic Control 27(4), 869-879.

Bekiaris-Liberis, N. and M. Krstic (2012). Compensation of time-varying input and state delays for nonlinear systems. Journal of Dynamic Systems, Measurement and Control 134(1), 1-14.

Bekiaris-Liberis, N. and M. Krstic (2013). Nonlinear Control Under Nonconstant Delays. SIAM. Philadelphia, PA.

Bresch-Pietri, D. and N. Petit (2014). Robust compensation of a chattering time-varying input delay. In Proceedings of the IEEE Conference on Decision and Control. Los Angeles, LA. pp. 457-462. 
Cacace, F., A. Germani and C. Manes (2014a). A chain observer for nonlinear systems with multiple timevarying measurement delays. SIAM Journal on Control and Optimization 52(3), 1862-1885.

Cacace, F., A. Germani and C. Manes (2014b). Exponential stabilization of linear systems with time-varying delayed state feedback via partial spectrum assignment. Systems and Control Letters 69, 47-52.

Cacace, F., A. Germani and C. Manes (2014c). Predictor-based control of linear systems with large and variable measurement delays. International Journal of Control 87(4), 704-714.

Cacace, F., F. Conte, A. Germani and G. Palombo (2016a). Optimal control of linear systems with large and variable input delays. Systems and Control Letters 89, 1-7.

Cacace, F., F. Conte, A. Germani and P. Pepe (2016b). Stabilization of strict-feedback nonlinear systems with input delay using closed-loop predictors. International Journal of Robust and Nonlinear Control 26(16), 3524-3540.

Cacace, F., F. Conte and A. Germani (2016c). Memoryless approach to the LQ and LQG problems with variable input delay. IEEE Transactions on Automatic Control 61(1), 216-221.

Fridman, E. and S-I. Niculescu (2008). On complete Lyapunov-Krasovskii functional techniques for uncertain systems with fast-varying delays. International Journal of Robust and Nonlinear Control 18(3), 364374.

Germani, A., C. Manes and P. Pepe (2002). A new approach to state observation of nonlinear systems with delayed output. IEEE Transactions on Automatic Control 47(1), 96-101.

Kamalapurkar, R., N. Fischer, S. Obuz and W. Dixon (2016). Time-varying input and state delay compensation for uncertain nonlinear systems. IEEE Transactions on Automatic Control 61(3), 834-839.

Karafyllis, I. and M. Krstic (2013). Stabilization of nonlinear delay systems using approximate predictors and high-gain observers. Automatica 49(12), 3623-3631.

Khalil, H. (2002). Nonlinear Systems, Third Edition. Prentice Hall. Upper Saddle River, NJ.

Krstic, M. (2009). Delay Compensation for Nonlinear, Adaptive, and PDE Systems. Birkhauser. Boston.

Léchappé, V. (2015). Predictive Control and Estimation of Uncertain Systems with an Input Delay. PhD Thesis, Ecole Centrale de Nantes. Nantes, France.

Malisoff, M. and F. Zhang (2015). Robustness of adaptive control under time delays for three-dimensional curve tracking. SIAM Journal on Control and Optimization 53(4), 2203-2236.

Mathematica (2015). The world's definitive system for modern technical computing. Accessed June 30, 2016, http://www.wolfram.com/mathematica/.

Mazenc, F. and M. Malisoff (2014). Local stabilization of nonlinear systems through the reduction model approach. IEEE Transactions on Automatic Control 59(11), 3033-3039.
Mazenc, F. and M. Malisoff (2016). New prediction approach for stabilizing time-varying systems under time-varying input delay. In Proceedings of the 55th IEEE Conference on Decision and Control. Las Vegas, NV. pp. 3178-3182.

Mazenc, F. and M. Malisoff (2017). Stabilization of nonlinear time-varying systems through a new prediction based approach. IEEE Transactions on Automatic Control. DOI: 10.1109/TAC.2016.2600500, to appear.

Mazenc, F., M. Malisoff and S-I. Niculescu (2014). Reduction model approach for linear time-varying systems with delays. IEEE Transactions on Automatic Control 59(8), 2068-2082.

Mazenc, F., M. Malisoff and Z. Lin (2008). Further results on input-to-state stability for nonlinear systems with delayed feedbacks. Automatica 44(9), 2415-2421.

Mazenc, F., S-I. Niculescu and M. Krstic (2012). Lyapunov-Krasovskii functionals and application to input delay compensation for linear time-invariant systems. Automatica 48(7), 1317-1323.

Merad, M., R. Downey, S. Obuz and W. Dixon (2016). Isometric torque control for neuromuscular electrical stimulation with time-varying input delay. IEEE Transactions on Control Systems Technology 24(3), 971-978.

Michiels, W. and S-I. Niculescu (2007). Stability and Stabilization of Time-Delay Systems. SIAM. Philadelphia, PA.

Najafi, M., S. Hosseinnia, F. Sheikholeslam and M. Karimadin (2013). Closed-loop control of dead time systems via sequential sub-predictors. International Journal of Control 86(4), 599-609.

Peuteman, J. and D. Aeyels (2002). Exponential stability of nonlinear time-varying dierential equations and partial averaging. Mathematics of Control, Signals, and Systems 15, 42-77.

Richard, J.-P. (2003). Time-delay systems: an overview of some recent advances and open problems. Automatica 39(10), 1667-1694.

Sharma, N., C. Gregory and W. Dixon (2011). Predictorbased compensation for electromechanical delay during neuromuscular electrical stimulation. IEEE Transactions on Neural Systems and Rehabilitation Engineering 19(6), 601-611.

Witrant, E., C. Canudas de Wit, D. Georges and M. Alamir (2007). Remote stabilization via communication networks with a distributed control law. IEEE Transactions on Automatic Control 52(8), 1480-1485.

Zhou, B. (2014a). Pseudo-predictor feedback stabilization of linear systems with time-varying input delays. Automatica 50, 2861-2871.

Zhou, B. (2014b). Truncated Predictor Feedback for Time-Delay Systems. Springer-Verlag. Berlin, Germany.

Zhou, B., Z. Lin and G-R. Duan (2012). Truncated predictor feedback for linear systems with long timevarying input delays. Automatica 48, 2387-2399. 